\title{
Poor Man's Cell-block Preparation, a Novel Idea which Can be Applied to Basic Health Care Facilities in Resource-poor Settings
}

\author{
Huzaifa Saleem ${ }^{1}$, Mehwish Javed ${ }^{2}$, Aniqua Saleem ${ }^{3}$, Sadia Atif ${ }^{4}$, Rabeea Rafi ${ }^{5}$
}

${ }^{1}$ Consultant Histopathologist, Pathology Department, Armed Forces Institute of Pathology, Rawalpindi, Pakistan. ${ }^{2}$ Consultant Histopathologist, Pathology Department, Railways Hospital, Rawalpindi Pakistan. ${ }^{3}$ Senior Registrar, Radiology Department, Rawalpindi Medical University, Rawalpindi, Pakistan. ${ }^{4}$ Consultant Histopathologist, Healthways Lab, Rawalpindi, Pakistan. ${ }^{5}$ Consultant Histopathologist, Pathology Department, Tehsil Head Quarter Hospital, Attock, Pakistan.

\begin{abstract}
Objective: To determine the diagnostic yield of cell blocks, prepared by the vapour fixation method, using laboratory supplies easily available at any low resource laboratory setup or outpatient department of a basic healthcare unit. Methods: Prospective descriptive study was carried out at Healthways Laboratories Rawalpindi, for 6 months from $1^{\text {st }}$ January 2017 to $30^{\text {th }}$ June 2017. Walk in patients referred to the lab for FNAC were selected by non probability convenient sampling. After preparing FNAC slides from first pass, the patients were briefed about the cell block material and after informed consent second dedicated passes were done to make cell blocks. Results: Out of 47 cases, there were $25(53.2 \%)$ breast lumps, 8 (17\%) superficial collections, 7 (15.1\%) lymphadenopathies, $6(12.8 \%)$ thyroid swellings and 1 salivary gland swelling. Technique yielded moderate to high cellularity in 33 (70.2\%) cases, 16 from malignant and 1 from benign breast lumps, 5 from thyroid, 3 from reactive and 3 from metastatic lymphnodes, 3 from abscesses, 1 from lipoma and 1 from salivary gland. Low cellularity $14(29.8 \%)$ cases were from 5 benign and 3 malignant breast lumps, 2 hematomas and 1 case each from thyroid, metastatic lymphnode, abscess and lipoma. Good cellularity was achieved in $16(84.2 \%)$ of 19 cases of malignant breast lesions, $3(15.8 \%)$ were hypo cellular of which 1 was non-diagnostic on FNAC smear. Cell block diagnosis was comparable to FNAC in 35 of 47 (74.4\%) cases. Non diagnostic cases on cell block were more, 13 versus 3 cases on FNAC. The kappa value of agreement in diagnosis was 0.64 . Conclusions: Poor man's cell block method is simplest and effective method of cell block preparation which must be advocated at basic healthcare units and low tech laboratory. Its diagnostic potential must be further explored.
\end{abstract}

Keywords: Poor man's cell block- biopsy- lymphnodes- thyroid- abscess- lipoma- salivary glands

\section{Introduction}

The cell block technique has been in use for over a hundred years. The idea was initiated by Bahrenburg (1896) that applied it to a large quantity of ascitic fluid from two patients and was able to convert the fluid specimen into histological sections. The technique has evolved and various methods of cell block preparations have emerged [1-2].

The college of American Pathologists' cytology committee reviewed available literature and published a recognition of about 8 to 9 different methods of cell block preparation [3]. These methods range from the simple clot and scrape method which employs scraping off the material from a pre-fixed smear and the low tech, low cost vapour fixation method to the highly sophisticated, fully automated cellient cell block technique Wagner DG et al., (2011) [4]. The present study helps to elucidate the role of cell block material as a better specimen for ancillary studies, with better options for optimal sampling and triage [3].

\section{Corresponding Author:}

Dr. Aniqua Saleem

Department of Radiology, District Head Quarters Hospital, Rawalpindi Medical University, Rawalpindi, Pakistan.

Email: aneeqa_068@hotmail.com 
The most common method employed for making cell block is the, 'Plasma Thrombin method' [3-5]. This technique offers valuable material for ancillary studies is low cost and can utilize fine needle aspirates as well as fluid specimens. However, the technique is slightly cumbersome and labour intensive. There is also a risk of cross contamination with plasma or thrombin. A technique was proposed by Mayall and Darlington (2010), which utilized the vapour fixation method of making cell block from fine needle aspirated material [6]. The technique which refers to as the poor man's cell block is simple and almost cost-free as no extra reagents or expensive laboratory equipment is required for the preparation of cell block. Despite being a very cost effective and a simpler technique, it is not widely used even in poor countries probably because very little data is available on the effectivity and efficiency of the technique and its ability to preserve cellular detail and antigenicity. The reason might be that the technique is less advocated, not very well known and sounds crude as it employs almost no specific reagents or equipment.

The role of cell blocks as an adjunct to fine needle aspiration cytology (FNAC) smear preparation was established long time ago but in era of molecular diagnostics and personalized medicine a morphological diagnosis alone does not suffice. Pathologists are now faced with more challenges and a steady increase is seen in the request for molecular testing on patient sample. Use of cytology material for ancillary tests is thus, being advocated more and more [7-9]. This becomes even more relevant in patients with high-stage, surgically unresectable malignant disease. In such cases smaller biopsies with minimal complications are desired [7]. Many studies in this regard are being carried out, mainly with the aim of validating the results of immunohistochemistry (IHC), fluorescence in situ hybridization and molecular genetics on cell block material [9-10]. Cell blocks are a preferred choice among cytology material for ancillary tests as they are known for their semblance to histological sections, yield formalin fixed paraffin embedded blocks, can be archived and multiple sections for multiple purposes are available. Since, most ancillary techniques have been validated on formalin fixed paraffin embedded material hence the preferred choice of fixative in cell block material is also formalin [10].

Although efforts are directed towards mutational analysis in cell block material, its importance as a cost effective technique with wider applicability cannot be undermined. In resource-poor countries, with low tech laboratory setups, un-affording patients and lack of qualified personnel, the novel ideas of personalized medicine is still a far- fetched dream On the contrary, there is need for cheaper and more accessible basic health care diagnostic services for all.

The purpose of this study was to test the diagnostic yield of cell blocks, prepared by the vapour fixation method, using laboratory supplies easily available at any low resource laboratory setup or the outpatient department of a basic health care unit. If the technique is found to offer good preservation of cellular details and IHC can be conveniently applied on the cell block prepared, then it can result in considerable benefit to the patient allowing diagnosis in a single test procedure. It will also minimize unnecessary delay in treatment as in developing countries most of the patients are lost on follow up because of financial constraints. This method could even be applied in centres which can afford equipment for cell block preparation by other methods in select cases. The cost effectiveness of the technique will lessen the financial burden on the patients. It may serve as an alternative to the surgical biopsy, which obviously has its own financial implications and associated risks and complications.

\section{Materials and Methods}

\section{Objective}

The study was carried out at Healthways Laboratories Rawalpindi, Pakistan, over a period of 6 months from 1st January 2017 to $30^{\text {th }}$ June 2017 . A sample size of 47 was calculated by nQuery Advisor sample size calculator by taking power of test as $90 \%$, to detect a difference in proportion of 0.3 when discordant pair is expected to be 0.5 and analyzed by McNemar's chi square test with 0.05 one sided significance and Kappa statistic. These patients were walk in patients referred to the lab for FNAC. There were no preset inclusion or exclusion criteria. The patients were included in the order they came to the lab. After preparing FNAC slides from first pass, the patients were briefed about the cell block material as an adjunct to diagnosis. Second dedicated passes were done to make cell blocks after informed consent was received. In a few cases, such as those with obvious clinically malignant breast lumps expected to yield good enough aspirate in one pass, informed consent was taken before hand. Complete contact information was taken from the patient and they were encouraged to share their biopsy report findings.

The Technique and Supplies used:

Mayall and Darlington (2013), the pioneers of the technique, expelled the contents of the needle on an inverted lid of a universal container to form a blob on the inside of the lid [6]. Next they pushed a tissue soaked in formalin into the container and closed it over the inverted lid. The inverted container was then left standing for 6 hours at room temperature for vapour fixation. This blob was then removed by flooding the lid with formalin. This breaks the limpet suction between the lid and the cell block material which is then easily removed, cassetted and sent to the histopathology lab in $10 \%$ buffered liquid formalin. In this study, we have used the same technique, but instead used a sterile urine sample collection bottle. First-aid bandage was used to hold cotton soaked in formalin at the base of the container. The contents of the needle were expelled onto inverted lid and the bottle with soaked cotton was closed over it. After 6 hours, the contents were transferred to a cassette and sent to the histopathology lab (Figure 1). The FNAC was done by a senior resident histopathologist. Slides of the cell block were viewed independently by two senior residents and one consultant histopathologist. The slides were assessed 
for cellularity and architecture. Cellularity was reported as acellular, poor, moderate or high. The architecture was reported as preserved or not preserved. FNAC smear diagnosis and cell block diagnosis were separately categorized as non-diagnostic, benign/reactive, atypical or malignant. The opinion of consultant histopathologist was considered as final.

\section{Results}

A total of 47 patients were included in the study. Most frequently encountered lumps were in breast, contributing $25(53.2 \%)$, followed by $7(15.1 \%)$ cases of lymphadenopathy and 6(12.8\%) cases of thyroid swellings. One case of salivary gland swelling presenting during study period was also included. Eight cases were from superficial abscesses, lipomas and hematomas (Table 1).

The technique yielded moderate to high cellularity in $33(70.2 \%)$ cases, while $14(29.8 \%)$ cases had unsatisfactory cellularity. Out of the 14 low cellularity cases, 5 were benign and 3 were malignant breast lumps, 2 were old haemorrhages, 1 was a case of thyroid, 1 of metastatic tumour in lymph node, 1 abscess and 1 lipoma. Out of 33 cases with moderate to high cellularity, 16 were from malignant breast lesions, 1 from benign breast lump, 5 from thyroid lumps, 3 from reactive lymph nodes, 3 from metastatic lymph nodes, 3 from abscesses, 1 from a lipoma and a single case from salivary gland aspirate. (Table 2).

Out of a total 19 cases of malignant breast lesions, good cellularity was achieved in $16(84.2 \%)$ cases and only $3(15.8 \%)$ were hypo cellular of which 1 was nondiagnostic on FNAC smear. Five out of $6(83.3 \%)$ thyroid lumps were cellular, 1 non cellular thyroid swelling was diagnosed as colloid cyst on FNAC as abundant colloid was visible on both smear and cell block. Lymph nodes, both reactive $(100 \%)$ and metastatic $(75 \%)$, yielded cellular cell blocks (Table 2). Diagnosis on cell block was comparable to diagnosis on FNAC in 35 of 47 (74.4\%) cases. Non diagnostic cases on cell block were more, 13 cases as opposed to only 3 undiagnosed cases on FNAC. The kappa value of agreement in diagnosis was 0.64. (Table 3).

Most of the non-diagnostic cell blocks were those

Table 1. Types of Specimen

\begin{tabular}{lc}
\hline Type of Specimen & Count $(\%)$ \\
\hline Malignant breast lesion & $19(40.4)$ \\
Benign breast lesion & $6(12.8)$ \\
Thyroid & $6(12.8)$ \\
Metastatic lymphnode & $4(8.5)$ \\
Reactive lymphnode & $3(6.4)$ \\
Salivary gland & $1(2.1)$ \\
Abscess/Inflammation & $4(8.5)$ \\
Hemorrhage/Hematoma & $2(4.3)$ \\
Lipoma & $2(4.3)$ \\
Total & $47(100)$ \\
\hline
\end{tabular}

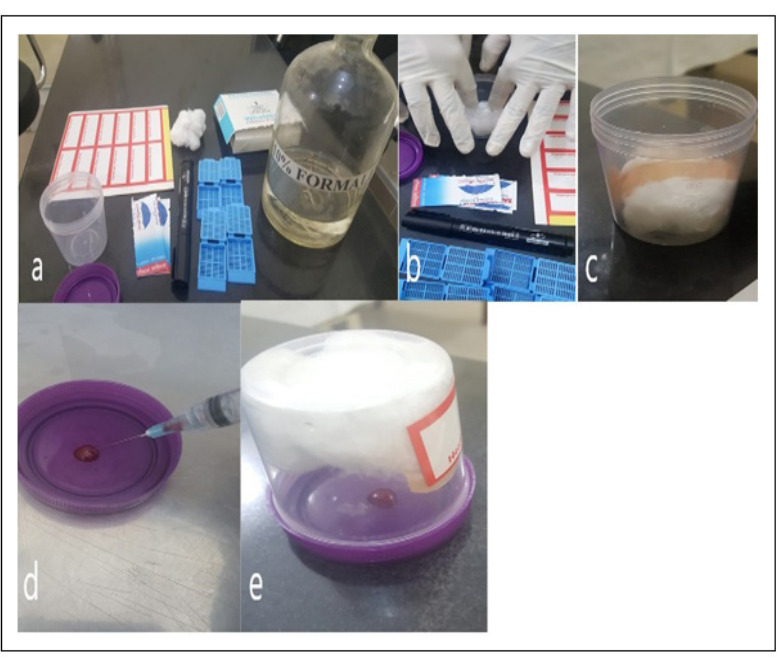

Figure 1. Method of Vapour Fixation; Figure Ia, Equipment required to produce a poor man's cell block; Figure Ib, tissue soaked in formalin; Figure Ic, First-aid bandage is used to hold cotton soaked in formalin at the base of the container; Figure Id, contents of the needle on an inverted lid; Figure Ie, container closed over the inverted lid

that were benign or reactive lesions. Despite fewer yields on aspirate and poor cellularity on cell blocks, diagnosis on smear was possible. On the other hand, all malignant lesions made good cellularity cell blocks with only a single case that was discrepant on FNA smear and constituent cell block.

\section{Discussion}

Following encouraging results in the work done on application of ancillary techniques on cell blocks, and some newer guidelines labelling them as preferred cytological specimen for optimal use in molecular tests, there are calls for reviewing the methods of preparation and optimizing the cellularity achieved $[2,5,9,10]$. Various researchers have developed their own methods for cell block processing. Most are aimed at better procurement of material, more cellularity and good preservation of cellular details and architecture [6, 11-14]. Others have compared two to three of these methods to validate the success of a particular technique [2, 4, 15]. The methods vary immensely in their methodology and technical details [7]. Some are simple but have inconsistent results. Others are complex, time consuming but worthwhile. Yet some are cumbersome and offer little added advantage.

In the earliest attempts of making cell blocks, simple sedimentation of cytological sample was used. This had unsatisfactory results with majority cases having low cellular yields. Later centrifugation was added with some success. Major impediment in preparation of a well preserved cellular cell block was the fixation of the sediment. Routine processing during dehydration and paraffin-embedding would result in significant disintegration of cellular fragments and inconsistent cellularity, with breaking up of sample into minute pieces and scattering of diagnostic material in various 
Table 2. Type of Specimen and Cellularity Attained (in percentage)

\begin{tabular}{|c|c|c|c|c|c|}
\hline \multicolumn{3}{|c|}{ Type of Specimen } & \multicolumn{2}{|c|}{ Cellularity } & \multirow[t]{2}{*}{ Total } \\
\hline & & & Acellular to poor & moderate to high & \\
\hline \multirow[t]{2}{*}{1.} & Malignant breast lesion & Count & 3 & 16 & 19 \\
\hline & & $\%$ within Type of Specimen & $15.80 \%$ & $84.20 \%$ & $100.00 \%$ \\
\hline \multirow[t]{2}{*}{2.} & Benign breast lesion & Count & 5 & 1 & 6 \\
\hline & & $\%$ within Type of Specimen & $83.30 \%$ & $16.70 \%$ & $100.00 \%$ \\
\hline \multirow[t]{2}{*}{3.} & Thyroid & Count & 1 & 5 & 6 \\
\hline & & $\%$ within Type of Specimen & $16.70 \%$ & $83.30 \%$ & $100.00 \%$ \\
\hline \multirow[t]{2}{*}{4.} & Metastatic lymphnode & Count & 1 & 3 & 4 \\
\hline & & $\%$ within Type of Specimen & $25.00 \%$ & $75.00 \%$ & $100.00 \%$ \\
\hline \multirow[t]{4}{*}{5.} & Reactive lymphnode & Count & 0 & 3 & 3 \\
\hline & & $\%$ within Type of Specimen & $0.00 \%$ & $100.00 \%$ & $100.00 \%$ \\
\hline & Salivary gland & Count & 0 & 1 & 1 \\
\hline & & $\%$ within Type of Specimen & $0.00 \%$ & $100.00 \%$ & $100.00 \%$ \\
\hline \multirow[t]{2}{*}{6.} & Abscess/Inflammation & Count & 1 & 3 & 4 \\
\hline & & $\%$ within Type of Specimen & $25.00 \%$ & $75.00 \%$ & $100.00 \%$ \\
\hline \multirow[t]{2}{*}{7.} & Hemorrhage/Hematoma & Count & 2 & 0 & 2 \\
\hline & & $\%$ within Type of Specimen & $100.00 \%$ & $0.00 \%$ & $100.00 \%$ \\
\hline \multirow[t]{2}{*}{8.} & Lipoma & Count & 1 & 1 & 2 \\
\hline & & $\%$ within Type of Specimen & $50.00 \%$ & $50.00 \%$ & $100.00 \%$ \\
\hline \multirow[t]{2}{*}{ Total } & & Count & 14 & 33 & 47 \\
\hline & & $\%$ within Type of Specimen & $29.80 \%$ & $70.20 \%$ & $100.00 \%$ \\
\hline
\end{tabular}

depths of the resultant cell block. To avoid this loss and fragmentation of valuable diagnostic material, various cellular adjuvants were added. Hence, which so ever method is employed the basic steps remain the same, with most important being the hardening of the cellular pellet and its transfer for routine histological processing [2]. The goal of various researchers is therefore directed at improving this basic step by use of varied adjuvants and modified collection techniques.

The most popular method, to date remains the plasma thrombin method [3, 5]. The method is successfully applied to all kinds of cytological samples; fluids as well as needle aspirates. To the best of our knowledge the method has been in use since 1970's [16]. The method uses needle aspirate and needle rinses by saline or Roswell Park Memorial Institute medium. After centrifugation plasma and thrombin are added to the sediment and sample quickly agitated to form a clot. The clot is then transferred to a cassette and sent for routine histological processing. Histo gel Technique involves rinsing in formalin and fixing the sediment in warmed histogel. Alternatively some use agar for compact sediment. Colloidan bag technique collects the sample rinsed in formalin in a conical glass tube filled and emptied to get a colloidan membrane bag. Following centrifugation the cell pellet along with the bag is submitted for the histopathological processing $[11,15]$. Tissue coagulum method has been advocated for ultrasound guided endobronchial aspirations, which uses the wire stylet, provided in the needle kit, to remove the aspirated material that is allowed to clot in the syringe. As the material exits the needle tip the needle is directed in circular motion to form a cone shaped coagulum on a piece of filter paper. It is proposed that use of stylet to push the coagulum is better than spraying the material which had already been diluted in saline by pushing the plunger [17].

One of the simplest techniques deserving mention is one that involves scraping off the material from pre fixed smear. This could be tricky, involves decover slipping of slides and hence variable cellularity is achieved. The technique has evolved and modified cytoscrape blocks have been advocated. One such technique involves

Table 3. Correlation of Diagnostic Categories on Conventional Cytology Smear and Cell Block (Agreement Matrix)

\begin{tabular}{lcccccc}
\hline Diagnostic Categories & & \multicolumn{2}{c}{ FNA Smear Diagnosis } & & Total \\
& & Benign/reactive & Malignant & Atypical & Non diagnostic & \\
\hline Cell Block Diagnosis & Benign/reactive & 12 & 0 & 0 & 0 & 12 \\
& Malignant & 0 & 19 & 0 & 0 & 19 \\
& Atypical & 0 & 0 & 3 & 0 & 3 \\
Total & Non-diagnostic & 7 & 1 & 2 & 3 & 13 \\
\hline
\end{tabular}


decover slipping and mounting the cytoscrape in water, followed by use of agarose gel for embedding and re embedding [12]. The modifiers of technique were able to diagnose 10 cases of lymph node FNAC and applied 7 to 8 immunohistochemistry markers on each prepared cellblock. They believe that by concentrating the cytoscrape material in a small spot the loss of material can be avoided and a relatively uniform cellularity is achieved at each level of the cell block section [12]. Interest in smears is for the obvious reasons that these can be readily assessed for adequacy, easy to use for PCR- based tests and provides highest quality DNA for reliable molecular analysis [8, 18] have also demonstrated that cell block adequacy for molecular tests could be improved by addition of the diff quick stained slide smear.

Apart from those discussed, various other methods employed include, cell pellet formation using alcohol or formalin, BBC cell block fixative method, Shandon cytoblock technique, the new fully automated cellient cell block and the method we employed, the alcohol or formalin vapour fixation method.

Although cell blocks have now been in use since a century but various investigators are still desirous for their optimum utility in diagnosis [9]. Various researchers have highlighted the issue of low cellular yields in cell blocks, regardless of the method employed. Crapanzo et al. (2014) via a survey pointed out that $44 \%$ of the respondents were in some way dissatisfied with their cell blocks [5]. Their results showed that most commonly used methods were Plasma thrombin method, histogel method and the cellient automated technique. They stressed the need for a better methodical approach for achieving enhanced cellularity on cell blocks. Regardless of the method employed, some have reported the no diagnostic cellular yield in as low as 4-6\% cases only, [19-20], while Knoepp and Roh (2013) report suboptimal cellularity in as high as $57 \%$ cases. Investigators have, however, demonstrated that the low cellular yield problem can be adequately dealt by better triage beginning at the start of the aspiration and through rapid on site evaluation of the aspirated sample. Saqi (2016) also proposed that sharing of good experiences from laboratories be initiated and encouraged. Close collaboration between the pathologist and interventionalists can definitely ensure optimum results [21-22].

Cell blocks are important cytological diagnostic adjuncts that not only improve the smear diagnosis but yield formalin fixed paraffin embedded tissue block for a variety of ancillary tests and archival material $[7,19,23]$. Recent studies have elucidated the role of cell blocks in providing a definitive diagnosis on cytological material and proclaim them better than surgical biopsies for ancillary techniques. Leni et al. (2015) evaluated cell block preparations from endoscopic ultrasoundguided-fine-needle-aspiration of gastrointestinal solid neoplastic lesions and they concluded that cell block is most important complement diagnostic material [24]. Various studies on evaluation of thyroid nodules by FNA conclude cell blocks to be effective in increasing the diagnostic accuracy, by application of different immune cyto chemical stains [25-27]. In molecular testing for ALK and EGFR in lung cancers they have been labeled the preferred cytology specimen according to guidelines from the College of American Pathologists, International Association for the Study of Lung Cancer, and Association for Molecular Pathology [10]. Various researches on molecular testing on lung cancer, using endobronchial ultrasound-guided transbronchial needle aspiration specimens, have validated the effectivity and satisfaction of the cell blocks [20-22, 28].

In today's era of personalized medicine and targeted therapy, pathologists are faced with ever increasing new challenges in the form of greater number and complexity of tests on limited patient samples. Since the role of these medicines become even more relevant in patients with surgically unresectable tumors, efforts are directed to gain maximum diagnostic information through minimally invasive procedures. FNA with clear cut advantages of rapid onsite evaluation, better options for triage and ability to sample deep seated lumps is preferable. Cell blocks are routinely prepared from fine needle aspirates and are an integral part of any cytopathology lab. While the diagnostic potential and applicability of cell blocks is both agreed upon and well established, standardized preparatory method with optimum quality is still desirous.

Many researchers have sought to compare different methods to date, but in reality a systematic review and comparison of the various methods of cell block preparation is unlikely to be reliable as methods vary immensely in their methodologies and type of fixatives used. Jain (2017) conducted a research using defined subjective criteria and compared cell block sections for cellularity, fixation artefacts, morphologic details, and crispness of immunohistochemical staining [29]. He concluded cellular yield was slightly less with vapourfixed cell blocks, it had more staining artefacts $(33.3 \%$ versus $20 \%$ ) and it was time consuming ( $6 \mathrm{~h}$ versus $1 \mathrm{~h}$ ) in comparison to conventional formalin-fixed cell blocks however there was no difference in the two techniques with respect to immunohistochemical staining.

In our study we used the cell block method for solid tumors only however recent study by Shidham (2020) suggested that this method is applicable to fat pad aspirate, bone marrow aspirate, effusion fluids, and other fluid such as various washings and urine with good diagnostic yield and research outcomes [30]. In addition, similar and comparable specimens from veterinary sciences could also be assessed with cell block prepared by vapour method.

The formalin vapour fixation method, popularly known as the poor man's cell block method is in our knowledge the simplest method and even to our surprise yielded results beyond expectations. The overall cellularity was seen in $70.2 \%$ cases. Although there was higher number of non diagnostic cellularity cases on cell blocks, 13 as opposed to only 3 undiagnosed cases on FNA smear, the technique was quite successful with thyroid lesions and malignant breast lumps. Considering the simplicity of the technique the results are definitely comparable to any other cell block technique. The technique being so simple, that it may be carried out at any basic healthcare unit. It 
is proposed that atleast for select cases the technique be advocated at local, rural basic health care setups, and resultant block transported to a histopathological lab for further sectioning and interpretation. Further ancillary techniques may then be carried out on the cell block and a definitive diagnosis of benign versus malignant can be offered after a single FNA procedure. This will definitely benefit the poor patient by cutting down the costs of surgical biopsy and of commute to tertiary care centres in larger cities. The time delay in cancer diagnosis could be shortened as cancer diagnosis being a multistep process, is frequently quoted as the reason for delay [31]. Patients lost to follow up, and faraway diagnostic centres are among other causes, which could be complied for.

Although, long proposed, this technique is not widely practiced. It is now suggested that this method of preparation of cell block should be promoted in remote areas. In a low resource country, there are basic health care units providing primary health care to a wide population and rarely slides are received from isolated somewhat better facilitated basic health care units for opinion. These are seldom of use as they suffer from poor preservation, drying artefacts and low cellularity. The idea is to train basic health care providers to take aspirate and save it as cell block material in specimen containers. These may then be later on transported and processed at tertiary hospital lab care facilities. Though the idea has not been put to practice, it is believed that in a country with high incidence of breast cancer and social stigmata associated with it, these cell blocks may be useful in programmes targeting early primary diagnosis. In low tech, low resource laboratory setups of poor GDP countries simple diagnostic tests with wider applicability must be encouraged. It is better to be able to do something, than not to do anything at all.

In conclusion, poor man's cell block method is simplest method of cell block preparation with quality comparable to most other methods. Its use must be advocated at basic healthcare units and low tech laboratory setups however the diagnostic potential of the said method must be further explored.

\section{Acknowledgements}

We are indebted to the participants for their important contributions in this study.

\section{Funding}

This study was not funded by any organization.

\section{Conflict of Interest}

The authors declare that they have no conflict of interest.

\section{References}

1. Bahrenburg L. On the diagnostic results of the microscopical examination of the ascitic fluid in two cases of carcinoma involving the peritoneum. Cleveland Med Gaz. 1896;11:2748.

2. Jain D, Mathur SR, Iyer VK. Cell blocks in cytopathology: a review of preparative methods, utility in diagnosis and role in ancillary studies. Cytopathology. 2014 08;:n/a-n/a. https:// doi.org/10.1111/cyt.12174

3. Rollins S, Russell D. Cytopathology in focus: Cell blocks: Getting the most from the least invasive method. CAP Today. 2017.

4. Wagner DG, Russell DK, Benson JM, Schneider AE, Hoda RS, Bonfiglio TA. Cellient ${ }^{\mathrm{TM}}$ automated cell block versus traditional cell block preparation: A comparison of morphologic features and immunohistochemical staining. Diagnostic Cytopathology. 2010 Oct 14;39(10):730-736. https://doi.org/10.1002/dc.21457

5. Crapanzano JP, Heymann JJ, Monaco S, Nassar A, Saqi A. The state of cell block variation and satisfaction in the era of molecular diagnostics and personalized medicine. CytoJournal. 201403 20;11:7. https://doi.org/10.4103/17426413.129187

6. Mayall F, Darlington A. The poor man's cell block. Journal of Clinical Pathology. 201007 29;63(9):837-838. https:// doi.org/10.1136/jcp.2010.078410

7. Kalhor N, Wistuba II. Perfecting the fine-needle aspirate cell block. Cancer Cytopathology. 2013 03;121(3):109-110. https://doi.org/10.1002/cncy.21284

8. Knoepp SM, Roh MH. Ancillary techniques on directsmear aspirate slides. Cancer Cytopathology. 201207 11;121(3):120-128. https://doi.org/10.1002/cncy.21214

9. Saqi A. The State of Cell Blocks and Ancillary Testing: Past, Present, and Future. Archives of Pathology \& Laboratory Medicine. 201608 24;140(12):1318-1322. https://doi. org/10.5858/arpa.2016-0125-ra

10. Lindeman NI, Cagle PT, Beasley MB, Chitale DA, Dacic S, Giaccone G, Jenkins RB, Kwiatkowski DJ, Saldivar J, Squire J, Thunnissen E, Ladanyi M. Molecular Testing Guideline for Selection of Lung Cancer Patients for EGFR and ALK Tyrosine Kinase Inhibitors: Guideline from the College of American Pathologists, International Association for the Study of Lung Cancer, and Association for Molecular Pathology. Journal of Thoracic Oncology. 2013 07;8(7):823859. https://doi.org/10.1097/jto.0b013e318290868f

11. Fahey C, Bedrossian UK. Collodion Bag: A Cell Block Technique for Enhanced Cell Collection. Laboratory Medicine. 199302 01;24(2):94-96. https://doi.org/10.1093/ labmed/24.2.94

12. Choi Y, Jakhongir M, Choi S, et al . High-quality cell block preparation from scraping of conventional cytology slide: a technical report on a modified cytoscrape cell block technique. Malays J Pathol. 2016;38(3):295-304.

13. Lindsey KG, Houser PM, Shotsberger-Gray W, Chajewski OS, Yang J. Young Investigator Challenge: A novel, simple method for cell block preparation, implementation, and use over 2 years. Cancer Cytopathology. $2016 \mathrm{Nov}$ 09;124(12):885-892. https://doi.org/10.1002/cncy.21795

14. La Fortune KA, Randolph ML, Wu HH, Cramer HM. Improvements in cell block processing: The Cell-Gel method. Cancer Cytopathology. 201701 31;125(4):267-276. https://doi.org/10.1002/cncy.21814

15. Balassanian R, Wool GD, Ono JC, Olejnik-Nave J, Mah MM, Sweeney BJ, Liberman H, Ljung B, Pitman MB. A superior method for cell block preparation for fine-needle aspiration 
biopsies. Cancer Cytopathology. 201604 22;124(7):508518. https://doi.org/10.1002/cncy.21722

16. Harris M. Cell block preparation. Three-percent bacterial agar and plasma-thrombin clot method. Cytotechnol. Bull. 1974;11:6-7.

17. Yung RCW, Otell S, Illei P, Clark DP, Feller-Kopman D, Yarmus L, Askin F, Gabrielson E, Li QK. Improvement of cellularity on cell block preparations using the socalled tissue coagulum clot method during endobronchial ultrasound-guided transbronchial fine-needle aspiration. Cancer Cytopathology. 2011 Dec 05;120(3):185-195. https:// doi.org/10.1002/cncy.20199

18. Harada S, Agosto-Arroyo E, Levesque JA, Alston E, Janowski KM, Coshatt GM, Eltoum IA. Poor cell block adequacy rate for molecular testing improved with the addition of DiffQuik-stained smears: Need for better cell block processing. Cancer Cytopathology. 201505 08;123(8):480-487. https:// doi.org/10.1002/cncy.21561

19. Gupta N, Sekar A, Rajwanshi A. Role of FNAC, fluid specimens, and cell blocks for cytological diagnosis of lung cancer in the present era. Journal of Cytology. 2015;32(4):217. https://doi.org/10.4103/0970-9371.171219

20. Hopkins E, Moffat D, Parkinson I, Robinson P, Jersmann H, Dougherty B, Birader MI, Francis K, Nguyen P. Cell block samples from endobronchial ultrasound transbronchial needle aspiration provide sufficient material for ancillary testing in lung cancer-a quaternary referral centre experience. Journal of Thoracic Disease. 2016 09;8(9):25442550. https://doi.org/10.21037/jtd.2016.08.74

21. Bulman W, Saqi A, Powell CA. Acquisition and Processing of Endobronchial Ultrasound-guided Transbronchial Needle Aspiration Specimens in the Era of Targeted Lung Cancer Chemotherapy. American Journal of Respiratory and Critical Care Medicine. 201203 15;185(6):606-611. https://doi. org/10.1164/rccm.201107-1199ci

22. Sung S, Crapanzano J, DiBardino D, et al. Molecular testing (MT) on endobronchial ultrasound (EBUS) fine needle aspirates (FNA): significance of appropriate specimen triage (ST) at the start of the procedure. Mod Pathol. 2016;29:118.

23. Cristo APD, Goldstein HF, Faccin CS, Maia AL, Graudenz MS. Increasing diagnostic effectiveness of thyroid nodule evaluation by implementation of cell block preparation in routine US-FNA analysis. Archives of Endocrinology and Metabolism. 2016 08;60(4):367-373. https://doi. org/10.1590/2359-3997000000180

24. Ieni A, Barresi V, Todaro P, Caruso RA, Tuccari G. Cell-block procedure in endoscopic ultrasound-guided-fine-needleaspiration of gastrointestinal solid neoplastic lesions. World Journal of Gastrointestinal Endoscopy. 2015;7(11):1014. https://doi.org/10.4253/wjge.v7.i11.1014

25. Das DK, Al-Waheeb SKM, George SS, Haji BI, Mallik MK. Contribution of immunocytochemical stainings for galectin-3, CD44, and HBME1 to fine-needle aspiration cytology diagnosis of papillary thyroid carcinoma. Diagnostic Cytopathology. 2013 Nov 22;42(6):498-505. https://doi.org/10.1002/dc.23062

26. Zhang X. Value of Molecular Tests in Cytologically Indeterminate Lesions of Thyroid. Archives of Pathology \& Laboratory Medicine. 2015 Dec 01;139(12):1484-1490. https://doi.org/10.5858/arpa.2014-0245-ra

27. Cristo APD, Goldstein HF, Faccin CS, Maia AL, Graudenz MS. Increasing diagnostic effectiveness of thyroid nodule evaluation by implementation of cell block preparation in routine US-FNA analysis. Archives of Endocrinology and Metabolism. 2016 08;60(4):367-373. https://doi. org/10.1590/2359-3997000000180

28. Billah S, Stewart J, Staerkel G, Chen S, Gong Y, Guo M.
EGFR and KRAS mutations in lung carcinoma. Cancer Cytopathology. 201103 11;119(2):111-117. https://doi. org/10.1002/cncy.20151

29. Jain E, Mohan H, Singhal N, Bisht B. The utility of vapor fixation for cell block preparation in fine needle aspiration of malignant lesions of lymph nodes. Journal of Histotechnology. 201701 02;40(1):2-8. https://doi.org/10. 1080/01478885.2016.1215624

30. Shidham VB. Specimen-specific cell-blocking approaches. Cytojournal. 2020 Dec 29;17:28. https://doi.org/10.25259/ cytojournal_75_2020

31. Lyratzopoulos G, Vedsted P, Singh H. Understanding missed opportunities for more timely diagnosis of cancer in symptomatic patients after presentation. British Journal of Cancer. 2015 03;112(S1):S84-S91. https://doi.org/10.1038/ bjc.2015.47

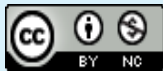

This work is licensed under a Creative Commons AttributionNon Commercial 4.0 International License. 